\title{
El rediseño del profesionalismo de las Ciencias de la Salud en el contexto post-pandemia de la COVID-19
}

The redesign of the Health Sciences' professionalism in the COVID-19 post-pandemic context

Filiación:

${ }^{1}$ Director de Docencia, Universidad Santa Paula, San José, Costa Rica. Posgrado en Salud Pública, Universidad de San Carlos,

Guatemala, Guatemala.

Correspondencia: $\square$ Jonnathan González Rodríguez, correo electrónico:jgonzalez@ uspsantapaula.com

Forma de citar: GonzálezRodríguez J. El rediseño del profesionalismo de las Ciencias de la Salud en el contexto post-pandemia de la COVID-19. Rev Ter. 2022;16(1): 4-7.

Palabras clave: profesionalismo, ciencias de la salud, educación superior

Key words: professionalism, health sciences, higher education

Financiamiento:

Ninguno

Conflictos de Interés:

Ninguno

Fecha enviado: 12 de enero del 2022.

Fecha aceptado: 21 de enero del 2022
La Conferencia Regional de Educación Superior de América Latina y el Caribe $(\mathrm{CRES})^{1}$, en su edición de 2018, planteó que la acreditación de alta calidad de carreras o instituciones no es el único e inequívoco parámetro para identificar si la forma en que se desarrolla el proceso educativo conduce a las personas hacia la excelencia. Esta premisa, no sólo es interesante, también es retadora.

A nivel internacional este planteamiento lleva años cimentándose en los escenarios más disruptivos e innovadores del ambiente universitario. Esto lo evidencia la transformación continua de las metodologías ligadas a los enfoques por competencias, la renovación de los mecanismos de desarrollo curricular de planes de estudio y el reciente interés internacional por centrar la mirada en el logro de habilidades o resultados de aprendizaje y ya no tanto en las capacidades de adquisición de conocimiento (como las asociadas a la memorización y al nivel académico como sinónimo de destreza $)^{2-5}$.

En el contexto de la pandemia por COVID-19, estas ideas se fortalecieron y generaron nuevos cuestionamientos acerca del impacto que la formación universitaria tiene para el contexto sociocultural: para la vida real donde las personas ejercen el quehacer especializado que han elegido como opción vocacional. Sin duda alguna los últimos años han comprobado que el sentido de una profesión -en armonía con el pensamiento de Viktor Frankl- radica en la capacidad que esta tiene para mejorar o potencializar el bienestar de las personas, en un entorno global, interdependiente y ecosistémico 5 .

Un proceso de formación o una oferta educativa que se preocupe exclusivamente por los factores internos de su puesta en marcha evidencia una práctica endogámica, que restringe los propósitos educativos a una mirada parcial e incompleta de la realidad a la que responderá el futuro ejercicio profesional. En respuesta a este riesgo, se han constatado múltiples esfuerzos por incluir en los modelos y sistemas de evaluación 
de la calidad educativa las capacidades que una institución tiene para incorporar lo más ágilmente posible las tendencias de la práctica profesional a la formación académica6-8.

Es fuera de las instituciones universitarias donde se desarrolla y consolida un ejercicio profesional, y aunque esto parezca lógico, deben reafirmarse los esfuerzos para que los procesos educativos sean armónicos con tal premisa. Hoy, por ejemplo, en las Ciencias de la Salud se ha renovado el interés por la comprensión del rol profesional en escenarios de evidentes riesgos epidemiológicos, así como también la priorización de la Salud Pública como concepto práctico y no como asunto accesorio a la labor clínica de atención de personas en los ámbitos institucionalizados del sistema sanitario; ambos temas anteriormente subvalorados en los catálogos de competencias deseables para personas graduadas de esta área de conocimiento.

Ahora, la pandemia también ha incidido en disciplinas como la Tecnología de Información y Comunicación, las Ingenierías y en las Ciencias Sociales, donde muchas Instituciones de Educación Superior ya viven el valor del desarrollo de conocimiento transdisciplinario en temas como el financiamiento de los servicios de salud, la demografía global, la gerontología social, las políticas humanitarias y la resolución de conflictos (como la pobreza extrema, el acceso a vacunas/ fármacos y la hambruna), la inequidad de acceso a la salud, la neuroepidemiología, el cambio climático y la salud sexual y reproductiva, entre otros (cf: líneas de investigación del Harvard School of Public Health en 2021) ${ }^{9}$.

De tal manera, no basta con ser tecnológicamente competente o con haber variado los canales de comunicación en el proceso educativo -incorporando herramientas de educación virtual- o en la atención de personas en los servicios de salud -incorporando herramientas de telemedicina- para considerarse a satisfacción con los cambios del contexto, sino que es necesario resignificar y rediseñar lo que se comprende como profesionalismo.

Un caso ejemplar de trabajo en pro de esta meta es lo actuado por las Ingenierías en sus procesos de formación. Dicha área de conocimiento, representada a nivel internacional por la Alianza Internacional de Ingeniería (IEA, por sus siglas en inglés) e impulsada en Costa Rica por el Colegio Federado de Ingenieros y Arquitectos (CFIA), así como por su Agencia de Acreditación de Programas de Ingeniería y Arquitectura (AAPIA), ha consensuado que para considerarse profesional en diversas ramas de la Ingeniería se deben de adquirir ciertas macro-habilidades a las que han denominado atributos de egreso. Estos atributos identifican metas de desempeño inherentes a los retos del quehacer profesional: análisis de problemas, investigación, habilidad de diseño, trabajo en equipo, habilidades de comunicación, ética y equidad, aprendizaje continuo y para la vida, habilidades de comunicación y profesionalismo, entre otras ${ }^{10-12}$.

Llamando la atención sobre este último atributo, el Canadian Engineering Accreditation Board (CEAB) describe al profesionalismo como "la comprensión de las funciones y responsabilidades (...) en la sociedad, especialmente el papel principal de protección del público y el interés público"11, lo que en un contexto post-pandemia implica la claridad del rol de la persona graduada en un contexto mediado por la incertidumbre, cumpliendo la obligación de proteger el interés superior de las personas a las que su quehacer impacta, de la procura de su bienestar y del análisis robusto de los determinantes que otorgan impacto y sentido a sus 
acciones disciplinares.

Este ejemplo, debe conducirnos a una profunda reflexión sobre qué tanto los procesos formativos en Ciencias de la Salud orientan a quienes los viven hacia un principio de realidad de la importancia e impacto de su futura actuación profesional, tanto en el ámbito personal como en el colectivo. De una manera más explícita, en este momento de la crisis sanitaria de salud, se debe propiciar la creación de vínculos estables entre la academia y la sociedad, como ya lo proponen las teorías de atención centrada en la persona y de calidad en los servicios (cf: International Society of Pshycooncology -IPOS- al respecto de la atención oncológica), entre otras que han reposicionado la ubicación de las personas en los modelos históricos de atención en Salud ${ }^{13,14}$.

La Comisión Económica para América Latina y el Caribe (CEPAL) ${ }^{15}$, ente especializado de las Naciones Unidas, ha reafirmado que la educación en tiempos de la pandemia debe incorporar tanto elementos característicos de la continuidad de aprendizajes y de priorización de los grupos vulnerables como la conciencia del impacto de la formación en el bienestar general de la población y desde esta perspectiva también resulta vital el cuestionamiento de cómo esta experiencia global acercará o distanciará a las personas graduadas de un auténtico profesionalismo.

Es imperativo que todas las personas vinculadas a la Educación Superior, tanto académicamente como en su gestión, evidencien claras intenciones de hacer que la formación universitaria trascienda lo evidente del otorgamiento de un título y les permita a quienes son profesionales ser artífices de un nuevo profesionalismo humanista y cada vez más capaz de reconocer a las personas, sus necesidades y retos dentro del abordaje de la
Salud ${ }^{16-18}$.

En el presente número de Revista Terapéutica se brindan evidencias sólidas de profesionales que han logrado trascender este reto de rediseño del ejercicio profesional, a través de la investigación robusta y científicamente válida, en los temas de dolor crónico, Esclerosis Lateral Amiotrófica, zika, aspectos de calidad en la regulación bioética y análisis de casos. Este recurso valioso, sin duda, conducirá al fortalecimiento de los espacios de desarrollo de las Ciencias de la Salud y al mejoramiento del bienestar de la población en general.

\section{Referencias Bibliográficas}

1. Conferencia Regional de Educación Superior de América Latina y el Caribe. Declaración de la III Conferencia Regional de Educación Superior para América Latina y el Caribe [Internet]. Córdoba, AR: UNESCO-IESALC; 2018. Disponible en: https://www.iesalc.unesco.org/wp-content/ uploads/2020/08/Declaracion2018PortFinal.pdf

2. Ampuero N, Báez M, Castillo C, Lagos J, Loncomilla $L$, Núñez $F$, et al. La evaluación progresiva del logro del perfil de egreso. En: Centro Interuniversitario de Desarrollo. Evaluación del logro de perfiles de egreso: experiencias universitarias [Internet]. Santiago: CINDA; 2017. 111-19. Disponible en: https://cinda.cl/wp-content/uploads/2017/07/ evaluacion-del-logro-de-perfiles-de-egreso-experiencias-universitarias.pdf

3. De Alba A. Currículum: crisis, mito y perspectivas. México: Editorial UNAM; 1991.

4. Díaz-Villa M. La flexibilidad curricular. Cali: Universidad de Cali; 2007.

5. Díaz-Villa M. Reforma curricular: elementos para el estudio de sus tensiones. En: Angulo Villa- 
nueva $\mathrm{R}$, Orozco Fuentes $\mathrm{M}$, coord. Alternativas metodológicas de intervención curricular en la educación superior. México: Plaza y Valdés; 2007. 6390

6. Agencia de Calidad de la Educación (CL). Evaluación progresiva. Santiago: Gobierno de Chile; 2018.

7. Riquelme-Bravo $P$, Ugüeño-Novoa $A$, Del Valle-Martín R, Jara Illanes E, Del Pino Sepúlveda M. Desafíos para la evaluación del perfil académico profesional, aproximaciones conceptuales. En: Centro Interuniversitario de Desarrollo. Evaluación del logro de perfiles de egreso: experiencias universitarias [Internet]. Santiago: CINDA; 2017. 19-37. Disponible en: https://cinda.cl/wp-content/ uploads/2017/07/evaluacion-del-logro-de-perfiles-de-egreso-experiencias-universitarias.pdf

8. Sistema Nacional de Acreditación de la Educación Superior (CR). Propuesta de nuevo modelo de acreditación. San José, CR: CONARE; 2018.

9. Harvard School of Public Health [Internet]. Boston: Harvard School of Public Health; 2022. Research Programs \& Projects; [aprox. 1 pantalla] Disponible en: https://www.hsph.harvard.edu/departments/

10. Colegio Federado de Ingenieros y Arquitectos, Agencia de Acreditación de Programas de Ingeniería y Arquitectura. Criterios y procedimientos de acreditación de programas de Ingeniería - 2018. San José, CR: CFIA; 2018.

11. Canadian Engineering Accreditation Board. 2017 Accreditation Criteria and Procedures [Internet]. Canadá: Engineers Canada; 2017. Disponible en: https://engineerscanada.ca/sites/default/files/ accreditation-criteria-procedures-2017.pdf

12. International Engineering Alliance. 25 Years. Washington Accord: 1989-2014. Celebrating international engineering education standards and re- cognition [Internet]. Wellington, NZ: International Engineering Alliance; 2014. Disponible en: https:// www.ieagreements.org/assets/Uploads/Documents/ History/25YearsWashingtonAccord-A5booklet-FINAL.pdf

13. Organización Mundial de la Salud. Informe sobre salud en el mundo: la atención primaria de la salud, más necesaria que nunca [Internet]. Ginebra: OMS; 2008. Disponible en: https://apps.who.int/iris/ bitstream/handle/10665/43952/9789243563732_ spa. pdf? sequence $=1 \&$ isAllowed $=y$

14. International Psycho-Oncology Society [Internet]. Toronto: IPOS; 2019. IPOS International Standard of Quality Cancer Care; [aprox. 1 pantalla]. Disponible en: https://ipos-society.org/about/quality

15. Comisión Económica para América Latina y el Caribe. La educación en tiempos de la pandemia de COVID-19 [Internet]. Santiago: CEPAL; 2020. Disponible en: https://repositorio.cepal.org/bitstream/handle/11362/45904/1/S2000510_es.pdf

16. Díaz-Villa M. El campo intelectual de la Educación en Colombia. Cali: Universidad de Cali; 1993.

17. Glazman-Nowalski R. Evaluación y exclusión en la enseñanza universitaria. México: Paidós; 2001.

18. Yzunza-Breña M. Perfil de egreso y formación profesional: una estrategia metodológica en el diseño curricular. México: Plaza y Valdés; 2010. 